\title{
Effect of Gender on the Pharmacokinetics of Eslicarbazepine Acetate (BIA 2-093), a New Voltage-gated Sodium Channel Blocker
}

\author{
Amílcar Falcão ${ }^{a}$, Joana Maia ${ }^{a}$, Luis Almeida ${ }^{a}$, Dago Mazur ${ }^{b}$, Manfred Gellert ${ }^{\mathrm{b}}$ and Patrício Soares-da-Silva ${ }^{\mathrm{a}, *}$ \\ ${ }^{a}$ Department of Research and Development, BIAL, 4745-457 S Mamede do Coronado, Portugal \\ ${ }^{\mathrm{b}}$ Scope International Life Sciences AG, Frohbösestraße 14, D-22525 Hamburg, Germany
}

\begin{abstract}
Purpose. To determine the effect of gender on the pharmacokinetics of eslicarbazepine acetate, a novel voltage-gated sodium channel blocker in the development for the treatment of epilepsy and bipolar disorder.

Methods. Single-centre, open-label, parallel-group study in 12 female and 12 male healthy subjects. The study consisted of a single-dose $(600 \mathrm{mg})$ period and a multiple-dose $(600 \mathrm{mg}$, oncedaily, for 8 days) period, separated by 4 days.

Results. Eslicarbazepine acetate was rapidly and extensively metabolized to eslicarbazepine (S-licarbazepine), the main active metabolite. Following a single-dose, arithmetic mean eslicarbazepine maximum plasma concentrations $\left(C_{\max }\right)$ and area under the plasma concentration-time curve over $24 \mathrm{~h}\left(A U C_{0-24}\right)$ and from 0 to infinity $\left(A U C_{0-\infty}\right)$ were, respectively, $9.3 \mu \mathrm{g} / \mathrm{ml}$, $128.5 \mu \mathrm{g} \mathrm{h} / \mathrm{ml}$ and $171.9 \mu \mathrm{g} \mathrm{h} / \mathrm{ml}$ in male subjects and $10.1 \mu \mathrm{g} / \mathrm{ml}, 150.1 \mu \mathrm{g} \mathrm{h} / \mathrm{ml}$ and $205.0 \mu \mathrm{g} \mathrm{h} / \mathrm{ml}$ in female subjects. At steady-state, $C_{\max }, A U C_{0-24}$ and $A U C_{0-\infty}$ were $15.5 \mu \mathrm{g} / \mathrm{ml}, 207.8 \mu \mathrm{g} \mathrm{h} / \mathrm{ml}$ and $295.8 \mu \mathrm{g} \mathrm{h} / \mathrm{ml}$ in male subjects, and $16.8 \mu \mathrm{g} / \mathrm{ml}, 214.5 \mu \mathrm{g} \mathrm{h} / \mathrm{ml}$ and $295.2 \mu \mathrm{g} \mathrm{h} / \mathrm{ml}$ in female subjects. Steady-state plasma concentrations were attained at 4 to 5 days of administration in both groups.

Eslicarbazepine $C_{\max }, A U C_{0-24}$ and $A U C_{0-\infty}$ female:male geometric mean ratios $(90 \% \mathrm{CI})$ were, respectively, $1.09(0.94 ; 1.24), 1.16(1.00 ; 1.33)$ and $1.17(0.99 ; 1.38)$ following single-dose, and 1.10 $(0.97 ; 1.25), 1.04(0.92 ; 1.17)$ and $1.01(0.88 ; 1.16)$ at steady-state.

Conclusion. At steady-state, the pharmacokinetic profile of eslicarbazepine acetate was not affected by gender. Copyright (C) 2007 John Wiley \& Sons, Ltd.
\end{abstract}

Key words: eslicarbazepine acetate; gender-effect; pharmacokinetics

\section{Introduction}

Eslicarbazepine acetate (BIA 2-093, S-(-)-10acetoxy-10,11-dihydro-5H-dibenz/b,f/azepine-5carboxamide) is a new voltage-gated sodium channel (VGSC) blocker currently being developed for the treatment of epilepsy and bipolar

*Correspondence to: Department of Research and Development, BIAL, À Av. da Siderurgia Nacional, 4745-457 S. Mamede do Coronado, Portugal. E-mail: Psoares.silva@bial.com disorder. It is chemically related to carbamazepine and oxcarbazepine, but has been specifically designed to avoid the production of toxic metabolites and to overcome enantiomeric impurity and the production of enantiomers or diastereoisomers of metabolites and conjugates without losing pharmacological activity [1].

Eslicarbazepine acetate metabolism varies significantly among different species. When a non-chiral method is used, the assay is not able to distinguish between eslicarbazepine [S-licarbazepine; (S)-(+)-10,11-dihydro-10-hydroxy-5H-dibenz/ 
b,f/azepine-5-carboxamide] and its R-enantiomer [(R-licarbazepine; (R)-(-)-10,11-dihydro-10hydroxy-5H-dibenz/b,f/azepine-5-carboxa-

mide], and the mixture is reported as BIA 2-005. In the rat, eslicarbazepine acetate is metabolized mainly to oxcarbazepine, BIA 2-005 being a minor metabolite. In the dog, BIA 2-005 and oxcarbazepine are the major metabolites. In the mouse, BIA 2-005 is the major metabolite. Following oral administration in humans, eslicarbazepine acetate is rapidly and extensively de-esterified by cytosol hydrolases to eslicarbazepine [2], the main active metabolite and plasma levels of parent drug (eslicarbazepine acetate) are usually undetectable in the plasma [2-4]. No cytochrome P450 enzymes are involved in the metabolism of eslicarbazepine acetate and it appears that eslicarbazepine acetate does not undergo auto-induction of its metabolism. Glucuronidation appears to be the main eslicarbazepine metabolic pathway.

Eslicarbazepine acetate and metabolites were tested in several models predictive of anticonvulsant efficacy. In the maximal electroshock seizure (MES) test in the rat, eslicarbazepine acetate was found to be equally potent to carbamazepine and more potent than oxcarbazepine, 2 and $4 \mathrm{~h}$ after the administration by gastric tube; $8 \mathrm{~h}$ after administration, the drugs were equipotent. Eslicarbazepine acetate and carbamazepine were similarly effective in preventing development of kindling seizures in kindled rats (data on file). A phase II placebo-controlled, randomized, parallel-group clinical trial in patients with partial epilepsy refractory to one or two standard anti-epileptic drugs showed that eslicarbazepine acetate $800 \mathrm{mg}$ once-daily is an effective and well-tolerated dose regimen [5]. Phase III clinical development for the adjunctive treatment of adult patients with partial epilepsy disorder is currently ongoing.

The prevalence of epilepsy is similar between males and females [6], and in clinical practice anticonvulsants are prescribed to both male and female patients. In 1993 the Food and Drug Administration (FDA) issued a guidance document requiring the inclusion of both genders in the clinical development of new drugs and in 1998 the FDA policy was confirmed by a report of the FDAMA (FDA Modernisation Act) Women and Minorities Working Group [7], requiring that new drug applications must include data on safety and effectiveness by sex. Gender-related differences in drug response can arise because of variation in pharmacokinetics or pharmacodynamics [8]. Although no gender-related differences have been reported with other VGSC inhibitors (e.g. oxcarbazepine and carbamazepine), this paper reports the results of an exploratory assessment of the effect of gender on the pharmacokinetic profile of eslicarbazepine acetate following a single dose and at steadystate of eslicarbazepine plasma concentrations. A primary analysis of this study showed that age has no significant effect on the pharmacokinetics of eslicarbazepine acetate and the results are published elsewhere [2].

\section{Methods}

\section{Study design}

This was an open-label, non-randomized, noncontrolled, parallel-group study performed at a single centre (Scope International Life Sciences AG, Hamburg, Germany). The trial consisted of one period (Phase A) during which participants were administered a single oral $600 \mathrm{mg}$ dose of eslicarbazepine acetate followed by a period (Phase B) during which participants were give a once-daily oral $600 \mathrm{mg}$ dose for 8 days (days 512). In Phase $A$, the subjects were admitted to the unit on day 0 (the day before the dosing) and remained under clinical supervision until at least $24 \mathrm{~h}$ post-dose (day 2); then, they left and attended the unit in the evening and the morning of days 3-5 for blood sampling and other study procedures. Phase B started following the $96 \mathrm{~h}$ post-dose procedures of Phase A (day 5) and subjects were requested to attend the unit in the morning of days 5-11 for pre-dose blood sampling for 'trough' eslicarbazepine plasma assay and administration of a $600 \mathrm{mg}$ dose of eslicarbazepine acetate. On the evening of day 11 subjects were admitted to the unit and the last dose of eslicarbazepine acetate was administered on the morning of day 12. Subjects remained under clinical supervision until the $24 \mathrm{~h}$ postdose procedures (day 13) and, then, they left and were requested to attend the unit in the evening 
of day 13 and in the morning of days 14, 15, 16 and 17 for blood sampling and other study procedures.

Eslicarbazepine acetate immediate release $600 \mathrm{mg}$ tablets manufactured by BIAL (S. Mamede do Coronado, Portugal) in accordance with Good Manufacturing Practice were used. On days 1 and 12 , participants were administered their $600 \mathrm{mg}$ dose between 0800 and $0900 \mathrm{~h}$ with $200 \mathrm{ml}$ of potable water. On those study days, participants fasted from at least $8 \mathrm{~h}$ before until $4 \mathrm{~h}$ post-dose.

The study was conducted according to the principles of the Declaration of Helsinki and the Good Clinical Practice. An Independent Ethics Committee (Ethik-Kommission der Ärztekammer Hamburg, Germany) reviewed and approved the protocol before the study started and all participants gave their written informed consent prior participation.

\section{Study population}

Participants enrolled were healthy male or female volunteers aged 18 to 40 years inclusive (young group) or 65 years or more (elderly), within 15\% (if young) or 20\% (if elderly) of ideal body weight, non-smokers or smokers of less than 10 cigarettes or equivalent per day. Female subjects were surgically sterilized or post-menopausal, or were required to be using doublebarrier or intrauterine device contraception methods. Subjects were required to abstain from taking any medication within 2 weeks prior admission and no concomitant medication was allowed during the study unless required for treatment of adverse events.

Twelve females [6 young (mean age \pm SD: $30.0 \pm 7.4$ years, range: $21-38$ years; weight $61.8 \pm 10.7 \mathrm{~kg}$, range: $48.0-73.0 \mathrm{~kg}$ ) and 6 elderly $(69.8 \pm 5.3$ years, range: $65-80$ years; weight $67.7 \pm 9.1 \mathrm{~kg}$, range: $55.0-82.5 \mathrm{~kg}$ )]; and 12 males [6 young ( $29.7 \pm 7.4$ years, range: $18-36$ years; weight $78.3 \pm 10.5 \mathrm{~kg}$, range: $66.0-98.0 \mathrm{~kg}$ ) and 6 elderly (70.0 \pm 6.0 years, range: $65-79$ years; weight $86.0 \pm 9.5 \mathrm{~kg}$, range: $76.0-103.5 \mathrm{~kg}$ )] completed the study according to the protocol.

\section{Plasma drug assay}

Blood samples ( $7 \mathrm{ml}$ of venous blood) were collected at the following times: pre-dose, and
$0.5,1,1.5,2,3,4,6,8,12,24,36,48,72$ and $96 \mathrm{~h}$ after the dose of eslicarbazepine acetate, on day 1 ; before the daily dose, on days 5-11 of Phase B; pre-dose, and $0.5,1,1.5,2,3,4,6,8,12,24,36,48$, 72,96 and $120 \mathrm{~h}$ post-dose, on day 12 . Blood samples were collected into tubes containing lithium heparin anticoagulant and centrifuged at approximately $1500 \mathrm{~g}$ for $10 \mathrm{~min}$ at $4^{\circ} \mathrm{C}$. The resulting plasma was separated into two equal aliquots of $1 \mathrm{ml}$ and stored at $-20^{\circ} \mathrm{C}$ until required for analysis.

Plasma concentrations of eslicarbazepine acetate were determined using isocratic liquid chromatography (LC) with single quadrupole mass spectrometric detection (MS).

The method involved the addition of $500 \mu \mathrm{l}$ of approximately $0.5 \mu \mathrm{g} / \mathrm{ml}$ of 10,11-dihydrocarbamazepine (internal standard prepared in acetonitrile:water, 3:97, v:v) to $250 \mu \mathrm{l}$ of plasma (centrifuged at $450 \mathrm{~g}$, prior to analysis) in a polypropylene tube. After vortex mixing for $10 \mathrm{~s}$, the mixture was transferred to a Schleicher and Schuell C18/100mg 96-well solid phase extraction plate. Each well was preconditioned with $800 \mu \mathrm{l}$ methanol, followed by $800 \mu \mathrm{l}$ acetonitrile and $800 \mu \mathrm{l}$ acetonitrile:water, 3:97, v:v, prior to application of the total sample volume. Each polypropylene tube was then washed with $500 \mu \mathrm{l}$ acetonitrile:water, $3: 97, \mathrm{v}: \mathrm{v}$ and the washings transferred to the respective well. The compounds were eluted into a collection plate with $750 \mu \mathrm{l}$ acetonitrile and the extract evaporated to dryness under oxygen-free nitrogen, at $40^{\circ} \mathrm{C}$. All solid phase extraction manipulations were undertaken using the Tomtec QUADRA $96{ }^{\mathbb{R}}$ Model 320 system and a vacuum was applied at each elution step. The final extract was reconstituted in $100 \mu \mathrm{l}$ of water:methanol (90:10, v:v) and mixed. The collection plate was then centrifuged at approximately $1000 \mathrm{~g}$ (at approximately $4^{\circ} \mathrm{C}$, for approximately $10 \mathrm{~min}$ ) prior to analysis. An aliquot of the final extract $(10 \mu \mathrm{l})$ was injected onto the LC-MS system.

The LC-MS system used in the analysis consisted of a Perkin Elmer series 200 micro pump, a Perkin Elmer series 200 autosampler, a Perkin Elmer/Sciex API 150EX single quadrupole mass spectrometer fitted with a Turbo IonSpray ${ }^{\circledR}$ source. Separation was achieved using a LichroCART 250-4 ChiraDex analytical 
column ( $\beta$-cyclodextrin, $5 \mu \mathrm{m})$, a LichroCART 4-4 ChiraDex guard column ( $\beta$-cyclodextrin, $5 \mu \mathrm{m}$ ), a Jones chromatography 7971 column heater at $50^{\circ} \mathrm{C}$, a mobile phase $\mathrm{A}(0.2 \mathrm{mM}$ sodium acetate, aq) and a mobile phase $B(0.2 \mathrm{mM}$ sodium acetate, $\mathrm{MeOH}$ ). The MS detector was operated in positive ion mode with mass transitions for eslicarbazepine and the internal standard of $277.08 \mathrm{amu}(200 \mathrm{~ms})$ and $261.05 \mathrm{amu}(200 \mathrm{~ms})$, respectively.

Calibration curves over the nominal concentration range $100-10000 \mathrm{ng} / \mathrm{ml}$ and a set of quality control (QC) samples (triplicates over 3 concentration levels: 200, 4000 and $8000 \mathrm{ng} / \mathrm{ml}$ ) were analysed with each batch of study samples. The QC samples were used to monitor the performance of the assay. The data for the QC samples showed that the overall imprecision of the method, measured by the coefficient of variation, ranged from $6.7 \%$ to $9.6 \%$. The mean accuracy ranged from $98.1 \%$ to $102.8 \%$ and the limit of quantification of the assay was $100 \mathrm{ng} /$ $\mathrm{ml}$.

Eslicarbazepine was synthesized in the Laboratory of Chemistry, BIAL, with a purity of $>$ 99.9\%. The internal standard, 10,11-dihydrocarbamazepine was supplied by Sigma-Aldrich (St Louis, MO).

\section{Pharmacokinetic and statistical evaluation}

The following eslicarbazepine pharmacokinetic parameters were derived from the individual plasma concentration-time profiles using noncompartmental analysis (WinNonlin, Version 4.0, Pharsight Corporation, Mountain View, CA): maximum observed plasma concentration $\left(C_{\max }\right)$, time of occurrence of $C_{\max }\left(t_{\max }\right)$, area under the plasma concentration versus time curve (AUC) from time zero to the last sampling time at which concentrations were at or above the limit of quantification $\left(A U C_{0-t}\right.$, calculated by the linear trapezoidal rule), $A U C$ over the $24 \mathrm{~h}$ dosing interval $\left(A U C_{\tau}\right), A U C$ from time zero to infinity $\left(A U C_{0-\infty}\right.$, calculated from $A U C_{0-t}+C_{\text {last }} / \lambda_{\mathrm{z}}$ where $C_{\text {last }}$ is the last quantifiable concentration and $\lambda_{\mathrm{z}}$ is the apparent terminal rate constant); $\lambda_{\mathrm{z}}$ was calculated by log-linear regression of the terminal segment of the plasma concentration versus time curve. The apparent terminal half-life

Copyright (C) 2007 John Wiley \& Sons, Ltd. $\left(t_{1 / 2}\right)$ was calculated from $\ln 2 / \lambda_{\mathrm{z}}$, the observed degree of accumulation $\left(R_{0}\right)$ was calculated from $A U C_{0-\infty}$ (day 12)/AUC ${ }_{0-\infty}$ (day 1) and the theoretical degree of accumulation $\left(R_{\mathrm{T}}\right)$ was calculated from $1 /\left(1-\exp ^{-\lambda} z^{\tau}\right)$, where $\tau$ is the dosing interval.

The rate and extent of systemic exposure to eslicarbazepine following single-dose and multiple-dose administration of eslicarbazepine acetate to female and male subjects was compared through the geometric mean ratios (GMR) and the corresponding $90 \%$ confidence intervals (90\% CI) of eslicarbazepine $C_{\max }, A U C_{\tau}(24 \mathrm{~h})$ and $A U C_{0-\infty}$. The adopted test procedure is analogous to equivalence testing. The pharmacokinetic parameters were logarithmically transformed prior to analysis. In accordance with the guidelines for bioequivalence testing, the bioequivalence can be assumed when the geometric mean ratio lies within the $90 \% \mathrm{CI}$ $0.80-1.25[9,10]$.

A $t_{\max }$ comparison between gender groups was performed assuming a non-parametric approach using the Wilcoxon signed rank test (untransformed values). All tests of significance were performed at the $p=0.05$ level.

In a previous study [11], reported intra-subject variability of $A U C$ and $C_{\max }$ was lower than $15 \%$. Assuming an intra-subject variability of $15 \%$, a GMR of 1.0 and a $\alpha=0.05$ (two-tailed), a sample size of $n=24$ subjects would provide at least $80 \%$ probability that the $90 \% \mathrm{CI}$ are contained within the interval commonly used for accepting bioequivalence $(0.80-1.25)[9,10]$.

All statistical analyses were performed using the statistical package SAS (Version 8.2, SAS Institute Inc, Cary, NC).

\section{Results}

Figures 1 and 2 display the mean eslicarbazepine concentration-time profiles in the male and female groups. The eslicarbazepine pharmacokinetic parameters in the male and female groups following a $600 \mathrm{mg}$ single-dose of eslicarbazepine acetate (Phase A) and following the last dose of a $600 \mathrm{mg}$ once-daily regimen for 8 days (Phase B) are presented in Table 1. The mean 'trough'

Biopharm. Drug Dispos. 28: 249-256 (2007) DOI: $10.1002 /$ bdd 

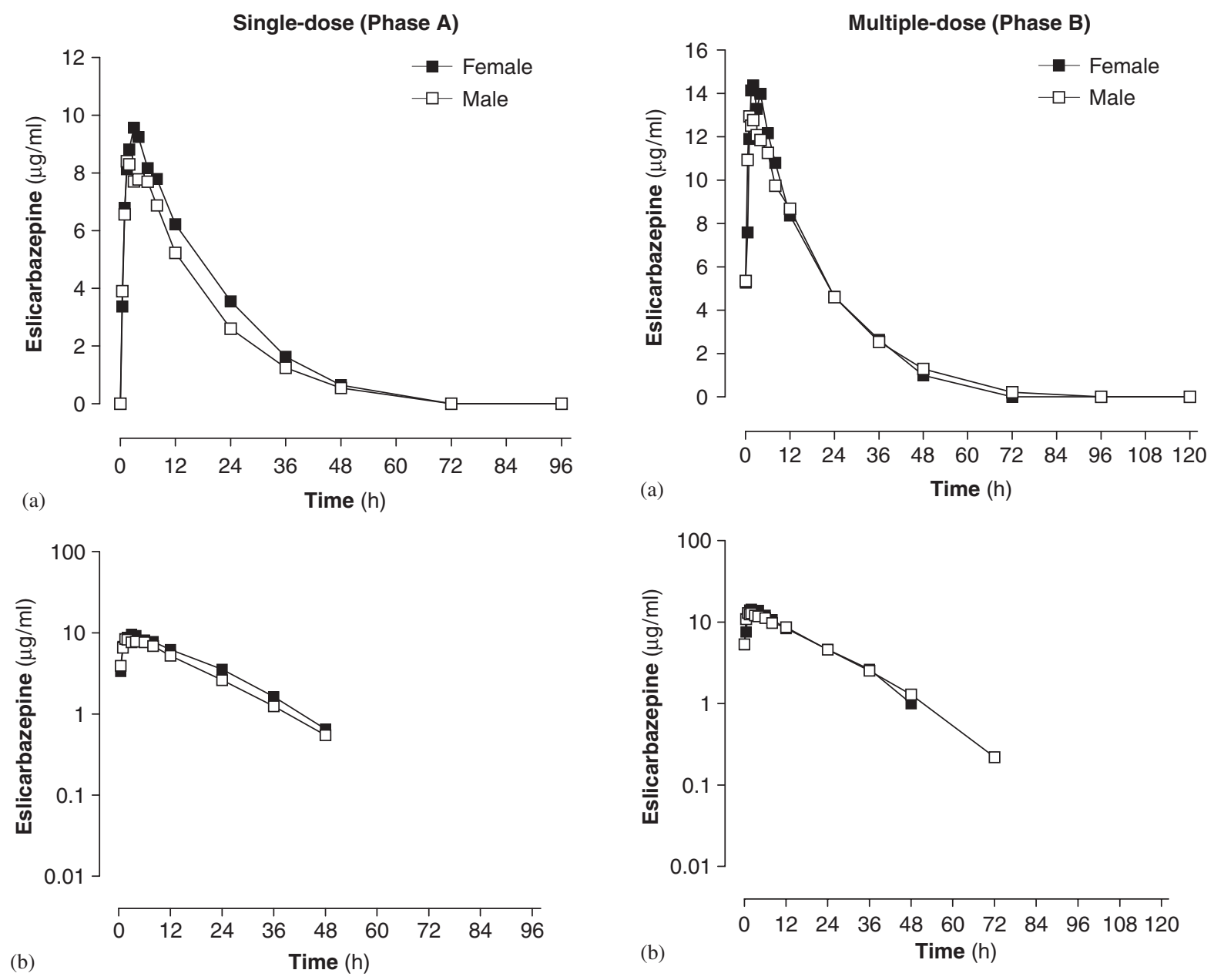

Figure 1. Mean eslicarbazepine plasma concentration-time profiles in the male and female groups following oral administration of a $600 \mathrm{mg}$ single-dose of eslicarbazepine acetate (Phase A) ( $n=12$ in each gender group): (a) linear scale and (b) semi-log scale

Figure 2. Mean eslicarbazepine plasma concentration-time profiles in the male and female groups following the last dose of a $600 \mathrm{mg}$ once-daily regimen of eslicarbazepine acetate for 8 days (Phase B) ( $n=12$ in each gender group): (a) linear scale and (b) semi-log scale

Table 1. Eslicarbazepine pharmacokinetic parameters following a single-dose of $600 \mathrm{mg}$ of eslicarbazepine acetate and following the last dose of a $600 \mathrm{mg}$ once-daily regimen of eslicarbazepine acetate for 8 days in the female and male groups $(n=12$ in each gender group)

\begin{tabular}{|c|c|c|c|c|}
\hline \multirow[t]{2}{*}{ Parameter } & \multicolumn{2}{|l|}{ Female } & \multicolumn{2}{|l|}{ Male } \\
\hline & Single-dose & Multiple-dose & Single-dose & Multiple-dose \\
\hline$C_{\max }(\mu \mathrm{g} / \mathrm{ml})$ & $10.1(2.3)$ & $16.8(2.0)$ & $9.3(1.7)$ & $15.5(3.7)$ \\
\hline$t_{\max }(\mathrm{h})$ & $3.0(2-6)$ & $2.0(1-6)$ & $2.0(1-6)$ & $1.3(1-4)$ \\
\hline$A U C_{\tau}(\mu \mathrm{gh} / \mathrm{ml})$ & $150.1(36.8)$ & $214.5(30.0)$ & $128.5(22.1)$ & $207.8(40.8)$ \\
\hline$A U C_{0-\infty}(\mu \mathrm{gh} / \mathrm{ml})$ & $205.0(67.5)$ & $295.2(44.4)$ & $171.9(34.2)$ & $295.8(65.0)$ \\
\hline$t_{1 / 2}(\mathrm{~h})$ & $10.3(0.95)$ & $10.4(1.0)$ & $10.7(1.1)$ & $11.2(1.8)$ \\
\hline
\end{tabular}

Results are expressed as arithmetic means with corresponding standard deviations in parentheses; $t_{\max }$ values are median with range values in parentheses.

Copyright (C) 2007 John Wiley \& Sons, Ltd. 
(pre-dose) concentrations during Phase B (days 5-12) are presented in Table 2.

Following a $600 \mathrm{mg}$ single-dose of eslicarbazepine acetate (Phase A), $C_{\max }$ of eslicarbazepine was reached $\left(t_{\max }\right)$ at $3.0 \mathrm{~h}$ (median) in the female group and $2.0 \mathrm{~h}$ in the male group. Thereafter, plasma concentrations declined in a multiphasic manner with a mean apparent $t_{1 / 2}$ of $10.3 \mathrm{~h}$ and $10.7 \mathrm{~h}$, respectively.

In Phase B (multiple-dose period), the steadystate of eslicarbazepine plasma concentrations was attained at 4 to 5 days of administration in both gender groups, consistent with an effective half-life in the order of $14.0 \mathrm{~h}$ (female) and $19.1 \mathrm{~h}$ (male), calculated from the theoretical degree of accumulation $\left(R_{\mathrm{T}}\right)$ where $\lambda_{\mathrm{z}}$ is the key value for its determination $\left(t_{1 / 2 \text { eff }}=\ln 2 / \lambda_{\mathrm{z}}\right)$ in accordance with Boxenbaum and Battle [12]. An observed accumulation factor $\left(R_{0}\right)$ of 1.44 (female) and 1.72 (male) was estimated.

Following the last dose of Phase $B, C_{\max }$ of eslicarbazepine was reached $\left(t_{\max }\right)$ at $2.0 \mathrm{~h}$ (median) in the female group and $1.3 \mathrm{~h}$ in the male group and, thereafter, plasma concentrations declined in a multiphasic manner with a $t_{1 / 2}$ of 10.4 and $11.2 \mathrm{~h}$, respectively.
The GMR and corresponding $90 \% \mathrm{CI}$ of eslicarbazepine $C_{\max }, A U C_{\tau}(24 \mathrm{~h})$ and $A U C_{0-\infty}$ are displayed in Table 3. Following single-dose administration of eslicarbazepine acetate, the $90 \% \mathrm{CI}$ for $A U C_{0-\infty}$ and $A U C_{\tau}$ are not contained within the acceptance $0.80-1.25$ interval $[9,10]$ and the extent of systemic exposure to eslicarbazepine following single-dose administration in female subjects can be assumed to be higher than that observed in male subjects. Following administration of eslicarbazepine acetate at steadystate, the $90 \%$ CIs are contained within the 0.80 1.25 interval for the parameters compared $\left(A U C_{0-\infty}, A U C_{\tau}\right.$ and $\left.C_{\max }\right)$ and, therefore, both rate and extent of systemic exposure to eslicarbazepine can be considered similar in both gender groups. The statistical power for $A U C_{0-\infty}, A U C_{\tau}$ and $C_{\max }$ was $0.85,0.92$ and 0.89 , respectively.

Eslicarbazepine apparent clearance $(C L / F=$ dose $(A U C)$ was higher in the female group when corrected for difference in body weight. Following eslicarbazepine acetate single-dose, a (CL/ F)/weight value of $48.6 \mathrm{ml} / \mathrm{h} / \mathrm{kg}$ and $44.1 \mathrm{ml} / \mathrm{h} /$ $\mathrm{kg}$ was obtained in the female and the male groups, respectively, corresponding to a female/

Table 2. 'Trough' (pre-dose) eslicarbazepine plasma concentrations during an oral $600 \mathrm{mg}$ once-daily regimen of eslicarbazepine acetate for 8 days in the male and female groups ( $n=12$ in each gender group)

\begin{tabular}{lllllllll}
\hline Group & \multicolumn{2}{l}{ Trough eslicarbazepine concentrations ${ }^{\mathrm{a}}(\mu \mathrm{g} / \mathrm{ml})$} & & & \\
\cline { 2 - 8 } & Day 5 & Day 6 & Day 7 & Day 8 & Day 9 & Day 10 & Day 11 & Day 12 \\
\hline Female & BLQ & $3.6(0.9)$ & $4.6(1.6)$ & $5.1(1.6)$ & $5.5(1.9)$ & $5.4(1.7)$ & $5.9(1.8)$ & $5.3(1.5)$ \\
Male & BLQ & $3.1(0.6)$ & $4.6(1.0)$ & $5.6(1.3)$ & $5.7(1.3)$ & $6.0(1.7)$ & $5.6(1.4)$ & $5.3(1.5)$ \\
\hline
\end{tabular}

Results are expressed as arithmetic means with corresponding standard deviations in parentheses. BLQ-below the limit of quantification of the assay $(0.1 \mu \mathrm{g} / \mathrm{ml})$.

${ }^{a}$ Repeated dose administration started at day 5.

Table 3. Eslicarbazepine geometric mean $C_{\max } A U C_{\tau}$ and $A U C_{0-\infty}$ and respective female:male ratios following a $600 \mathrm{mg}$ singledose and a $600 \mathrm{mg}$ once-daily regimen of eslicarbazepine acetate for 8 days ( $n=12$ in each gender group)

\begin{tabular}{llcccc}
\hline & & Female group & Male group & Ratio female/male & $90 \% \mathrm{CI}$ \\
\hline$C_{\max }(\mu \mathrm{g} / \mathrm{ml})$ & Single-dose & 9.9 & 9.1 & 1.09 & $0.94 ; 1.24$ \\
& Multiple-dose & 16.7 & 15.2 & 1.10 & $0.97 ; 1.25$ \\
$A U C_{\tau}(\mu \mathrm{g} \mathrm{h} / \mathrm{ml})$ & Single-dose & 146.5 & 126.7 & 1.16 & $1.00 ; 1.33$ \\
& Multiple-dose & 249.9 & 204.1 & 1.04 & $0.92 ; 1.17$ \\
$A U C_{0-\infty}(\mu \mathrm{g} \mathrm{h} / \mathrm{ml})$ & Single-dose & 197.2 & 168.8 & 1.17 & $0.99 ; 1.38$ \\
& Multiple-dose & 292.0 & 289.0 & 1.01 & $0.88 ; 1.16$ \\
\hline
\end{tabular}


male ratio of 1.10. At steady-state, $(C L / F) /$ weight was respectively $32.5 \mathrm{ml} / \mathrm{h} / \mathrm{kg}$ and $26.1 \mathrm{ml} / \mathrm{h} /$ $\mathrm{kg}$, with a female/male ratio of 1.25.

No statistical differences between gender groups were found in $t_{\max }$, following single-dose $(p=0.153)$ and multiple-dose $(p=0.085)$ administration.

\section{Discussion and Conclusion}

In previous studies eslicarbazepine acetate was shown to be extensively metabolized to eslicarbazepine and plasma concentrations of parent drug were systematically found to be below the limit of quantification [2-4]. Data from that study showed that age has no significant effect on the pharmacokinetics of eslicarbazepine acetate [2]. The present study reports further analyses on the effect of gender on the pharmacokinetics of this new VSGC inhibitor.

Failure to analyse gender-related differences in pharmacokinetics, adverse event profiles and efficacy of new medications limit the generalizability of such data to women. Genderrelated behaviour regarding drug disposition is an important issue in epilepsy and bipolar disorder, indications in which eslicarbazepine acetate is being developed, because women represent at least half of these patient populations [6,13]. Evidence of gender-related differences in pharmacokinetics abounds, but the mechanisms involved are complex and often poorly understood. The mechanistic processes underlying gender-specific pharmacokinetics can be divided into molecular and physiological factors [14]. Molecular factors include drug transporters and drug-metabolizing enzymes and the physiological factors include the lower weight and organ size, higher percentage of fat, lower glomerular filtration rate and different gastric motility in women compared with men [14].

In the present study, the results of single-dose and steady-state pharmacokinetics in 12 female and 12 male subjects were compared. Each gender group was composed of 6 young and 6 elderly subjects. Female and male groups did not differ regarding mean age $(49.9 \pm 21.7$ years and $49.8 \pm 22.0$ years, respectively) but, as expected, a difference was found in their mean weights $(64.7 \pm 9.9 \mathrm{~kg}$ and $82.2 \pm 10.4 \mathrm{~kg}$, respectively). This $21.3 \%$ lower weight in females could justify a corresponding difference in the main pharmacokinetic parameters assessed. Following singledose and multiple-dose, the rate of systemic exposure to eslicarbazepine, as assessed by $C_{\max }$, was $9 \%$ and $10 \%$ higher in females when compared with males (GMR 1.09 and 1.10, respectively). Using the bioequivalence approach, the $90 \% \mathrm{CIs}$ fall within the acceptance interval $0.80-1.25$. The extent of systemic exposure to eslicarbazepine, as assessed by $A U C_{\tau}$ and $A U C_{0-\infty}$, was $16 \%$ and $17 \%$ higher following single dose and $4 \%$ and $1 \%$ higher following multiple dose, in females when compared with males. Using the equivalence approach, the $90 \%$ CI fall within the acceptance interval 0.80 1.25 following administration at the steady state, but do not fulfil the bioequivalence criteria following single-dose. However, the product is aimed to be used chronically and, therefore, the relatively slight (although statistically significant) gender difference following single-dose is not foreseen to be of clinical significance.

Differences in absorption, distribution, metabolism and excretion may account for genderrelated pharmacokinetic differences [14]. In the present study, the time to maximum plasma eslicarbazepine concentration occurred later in women than men (although the difference did not attain statistical significance), which is in line with the reported slower gastric emptying observed in females when compared with male subjects [14].

A faster apparent clearance in women in comparison with men (female/male ratio of 1.25 , at steady-state) was reported in this study. The main metabolic steps involved in the metabolism of eslicarbazepine acetate and eslicarbazepine are, respectively, de-esterification by cytosol hydrolases (mainly in the liver) and glucuronidation by uridine 5-diphosphate glucuronosyltransferase (UGT). A faster eslicarbazepine glucuronidation in women is not expected to occur because UGT activity has been suggested to be lower in women compared with men [14]. Therefore, it is not expected that differences in de-esterification and subsequent glucuronidation of eslicarbazepine acetate may account for the

Biopharm. Drug Dispos. 28: 249-256 (2007) DOI: $10.1002 /$ bdd 
faster eslicarbazepine clearance observed in women.

Glomerular filtration rate is usually $10 \%$ lower in women than men and gender-related differences in tubular secretion and tubular reabsorption may occur, but have not been well characterized [14]. Non-clinical data have suggested that eslicarbazepine may undergo tubular reabsorption [data on file]. It might be hypothesized that a lower tubular reabsorption rate could be responsible for an apparently higher clearance in women, but differences between males and females at this level have not yet been evaluated.

Although a slight trend for higher values in the pharmacokinetic parameters related to systemic exposure were reported in the female group compared with the male group, differences were not proportional to the $21.3 \%$ lower body weight in females. The faster apparent clearance in women in comparison to men may compensate, at least partially, the lower body weight effect on the pharmacokinetic parameters, resulting in relatively similar values in both genders. No pharmacokinetic gender-related differences were observed with oxcarbazepine, a compound chemically related to eslicarbazepine acetate [15].

In conclusion, gender-related differences in systemic exposure to eslicarbazepine were slight and non-significant and unlikely to be relevant in clinical practice and can be explained by the demographic characteristics such as the higher mean weight observed in the male group in comparison with the female group. Therefore, dose-adjustments of eslicarbazepine acetate based on gender will not be required.

\section{References}

1. Benes J, Parada A, Figueiredo AA, et al. Anticonvulsant and sodium channel-blocking properties of novel 10,11dihydro-5H-dibenz[b,f]azepine-5-carboxamide derivatives. J Med Chem 1999; 42: 2582-2587.
2. Silveira P, Falcão A, Almeida L, Maia J, Soares-da-Silva P. BIA 2-093 pharmacokinetics in healthy elderly subjects. Epilepsia 2004; 45(Suppl. 3): 157.

3. Almeida L, Soares-da-Silva P. Safety, tolerability and pharmacokinetic profile of BIA 2-093, a novel putative anti-epileptic agent, during first administration to humans. Drugs R D 2003; 4: 269-284.

4. Almeida L, Soares-da-Silva P. Safety, tolerability, and pharmacokinetic profile of BIA 2-093, a novel putative antiepileptic, in a rising multiple-dose study in healthy humans. J Clin Pharmacol 2004; 44: 906-918.

5. Maia J, Almeida L, Soares-da-Silva P. BIA 2-093 as add-on therapy for refractory partial epilepsy in adults. Epilepsia 2004; 45(Suppl. 3): 158.

6. Gaitatzis A, Carroll K, Majeed A, Sander JW. The epidemiology of the comorbidity of epilepsy in the general population. Epilepsia 2004; 45: 1613-1622.

7. FDAMA Women and Minorities Working Group Report, Rockville (MD). US Department of Health and Human Services, Food and Drug Administration, July 1998.

8. Gandhi M, Aweeka F, Greenblatt RM, Blaschke TF. Sex differences in pharmacokinetics and pharmacodynamics. Annu Rev Pharmacol Toxicol 2004; 44: 499-523.

9. Center for Drug Evaluation and Research (CDER), Rockville (MD). Bioavailability and Bioequivalence Studies for Orally Administered Products-General Considerations. US Department of Health and Human Services, Food and Drug Administration, October 2000.

10. Committee for Proprietary Medicinal Products (CPMP). Note for Guidance on the Investigation of Bioavailability and Bioequivalence. CPMP/EWP/1401/98, The European Agency for the Evaluation of Medicinal Products (EMEA), July 2001.

11. Maia J, Vaz-da-Silva M, Almeida L, et al. Effect of food on the pharmacokinetic profile of eslicarbazepine acetate (BIA 2-093). Drugs R D 2005; 6: 201-206.

12. Boxenbaum $H$, Battle $M$. Effective half-life in clinical pharmacology. J Clin Pharmacol 1995; 35: 763-766.

13. Scully PJ, Owens JM, Kinsella A, Waddington JL. Schizophrenia, schizoaffective and bipolar disorder within an epidemiologically complete, homogeneous population in rural Ireland: small area variation in rate. Schizophr Res 2004; 67: 143-155.

14. Meibohm B, Beierle I, Derendorf H. How important are gender differences in pharmacokinetics. Clin Pharmacokinet 2002; 41: 329-342.

15. Van Heiningen PNM, Eve MD, Oosterhuis B, et al. The influence of age on the pharmacokinetics of the antiepileptic agent oxcarbazepine. Clin Pharmacol Ther 1991; 50: $410-419$. 